\title{
Active Surveillance for Thyroid Cancer: A Qualitative Study of Barriers and Facilitators to Implementation
}

Catherine B. Jensen

University of Wisconsin School of Medicine and Public Health, Madison, Wisconsin

Megan C. Saucke

Wisconsin Surgical Outcomes Research Program, Department of Surgery, University of Wisconsin

School of Medicine and Public Health, Madison, Wisconsin

Susan C. Pitt ( $\sim$ pitt@surgery.wisc.edu )

Division of Endocrine Surgery, Department of Surgery, University of Wisconsin School of Medicine and Public Health, Madison, Wisconsin

\section{Research Article}

Keywords: thyroid cancer, active surveillance, implementation science, qualitative, barriers, facilitators

Posted Date: March 11th, 2021

DOI: https://doi.org/10.21203/rs.3.rs-239823/v1

License: (1) This work is licensed under a Creative Commons Attribution 4.0 International License. Read Full License 


\section{Abstract}

Background: The 2015 American Thyroid Association guidelines supported active surveillance (AS) as a strategy for managing select low-risk thyroid cancers. Data examining physicians' attitudes about the acceptability of this option are limited. This study aimed to characterize the barriers and facilitators to implementing AS as perceived by practicing endocrinologists and surgeons in the United States.

Methods: We conducted 24 semi-structured interviews probing physicians' attitudes toward AS for patients with small, low-risk thyroid cancer. We used deductive content analysis guided by a well-known model of guideline implementation. Analysis characterized concepts and themes related to AS implementation as physician, guideline, or external factors. We performed member checking to validate results.

Results: The most prominent barriers to AS were related to physician factors, although guideline-specific and external barriers were also observed. Physician attitudes towards AS comprised the majority of physician-related barriers, while lack of knowledge about the guideline was also discussed. Participants' concerns about the potential negative outcomes resulting from observing a cancer were notable as were the lack of confidence in performing and offering surveillance. Beliefs about patient expectations and lack of knowledge about the guideline were also identified as barriers to offering surveillance. Guidelinespecific and external barriers included the vagueness of surveillance protocols, lack of data supporting active surveillance, and societal beliefs about cancer. Facilitators of active surveillance included patients' desire to avoid surgery and shared decision-making.

Conclusions: Barriers and facilitators of active surveillance for low-risk thyroid cancers exist at multiple levels. Strategies to increase adoption of active surveillance should focus on physicians' attitudes, patient expectations, data supporting surveillance outcomes, and promoting societal-level acceptance of surveillance.

\section{Background}

Active surveillance of low-risk thyroid cancer is a newer alternative approach to immediate surgery that has not been widely accepted in the United States (US). In 2015, the American Thyroid Association (ATA) guidelines supported active surveillance for select patients, such as those with $\leq 1 \mathrm{~cm}$ papillary thyroid cancer. ${ }^{1}$ This change was based on data from Japan showing low rates of disease progression while surveilling these small cancers. ${ }^{2,3}$ Since the guideline change, studies from other countries have also supported this approach. ${ }^{4-7}$ While prospective trials are underway in the United States (US) ${ }^{8,9}$, data remain limited, and as with any new approach, barriers to implementation exist. ${ }^{10-12}$

This qualitative study was designed to take an in-depth examination of barriers and facilitators to active surveillance in the US. Current data on physicians' attitudes towards active surveillance in the US are primarily based on surveys and have not to the best of our knowledge employed a qualitative 
approach. ${ }^{13-15} \mathrm{~A}$ clear, comprehensive understanding of barriers to active surveillance is necessary for developing and implementing effective strategies that increase use of this approach. Because overtreatment of patients with small, low-risk thyroid cancer remains a significant concern, increased use of active surveillance has the potential to reduce the resulting morbidity. ${ }^{16-19}$

\section{Methods}

\section{Study Population and Recruitment}

To characterize the barriers and facilitators of active surveillance for low-risk thyroid cancer, we conducted semi-structured interviews with actively practicing thyroid surgeons and endocrinologists in the US. Participants were identified and recruited at the 86th Annual ATA meeting as previously described. ${ }^{16}$ The Institutional Review Board approved this study (No. 2016 - 0884). Participants provided written informed consent to participate in this study which included the publication of anonymized direct quotations from interviews for publication.

\section{Data Collection}

Interviews were performed by two researchers with advanced training in qualitative interviewing (MCS, EMW). The interview guide was developed in conjunction with qualitative research experts and key stakeholders, including the target audience and thyroid cancer survivors, and piloted. The guide included a case-based clinical vignette and open-ended questions such as:

- What is your response to the ATA guideline that endorses active surveillance?

- What is your current approach to active surveillance?

- What concerns do you have about active surveillance?

- How did you implement active surveillance in your practice?

\section{Analysis}

Interviews were audio-recorded, transcribed, and deidentified. NVivo 11 (QSR International) was used for data management. To create the initial codebook, interview transcripts $(n=3)$ were analyzed using open coding by a team with diverse backgrounds in sociology (MCS), thyroid surgery (SCP), and population health (JLJ). As coding continued, emergent themes were incorporated, and taxonomy revised using constant comparative analysis. We resolved discrepancies between coders through discussion and group consensus.

Higher-level analysis of barriers and facilitators involved a deductive strategy to characterize and map all codes and themes onto Fischer's Guideline Implementation Framework. ${ }^{20}$ We selected this framework because it builds on Cabana's well-known model and identifies strategies to address each barrier. ${ }^{21}$ 
Fischer's framework differentiates barriers and facilitators into three levels: physician-related (personal), guideline-related, and external factors (Fig. 1).

\section{Member Checking}

Because initial data collection occurred within a year of the ATA guidelines release, we performed member checking in August-September 2020 to ensure the credibility and applicability of results. ${ }^{22}$ We emailed all participants a detailed summary of the results and asked about current relevance and emergence of new barriers or facilitators. Sixteen responded; all but one confirmed that the results resonated with their experiences and no new barriers or facilitators had developed in their practice since the initial interviews. Appropriate revisions were made based on the feedback.

\section{Results}

Twenty-four physicians including 12 surgeons and 12 endocrinologists participated in semi-structured interviews about active surveillance of low-risk thyroid cancer. Participants had a median age of 43 years (range 34-68) (Table 1). The majority were male (71\%), white (88\%), and in academic practice (88\%). Most participants $(67 \%)$ saw at least 10 patients/year with $\leq 1 \mathrm{~cm}$ thyroid cancers. To protect anonymity, quotes are identified as "surgeon" or "endocrinologist." 
TABLE 1. DEMOGRAPHIC DATA

\begin{tabular}{|c|c|c|}
\hline & $\begin{array}{l}\text { Surgeons } \\
\mathrm{n}(\%)\end{array}$ & $\begin{array}{l}\text { Endocrinologists } \\
\mathrm{n}(\%)\end{array}$ \\
\hline Age (median, range) & $41(34-67)$ & $47(35-68)$ \\
\hline Female & $2(18)$ & $5(42)$ \\
\hline Caucasian & $11(92)$ & $10(83)$ \\
\hline Academic practice & $11(92)$ & $10(83)$ \\
\hline \multicolumn{3}{|l|}{ Location } \\
\hline East/Northeast & $4(36)$ & $6(42)$ \\
\hline South & $2(18)$ & $0(0)$ \\
\hline Midwest & $2(18)$ & $4(33)$ \\
\hline West & $2(18)$ & $1(8)$ \\
\hline Treat $>10$ PTMC/year & $8(67)$ & $8(67)$ \\
\hline$>50 \%$ of practice is thyroid cancer & $12(100)$ & $6(50)$ \\
\hline$>25$ thyroid surgeries/year & $12(100)$ & - \\
\hline Member of the ATA & $12(100)$ & $12(100)$ \\
\hline Read all of 2015 ATA guidelines & $7(58)$ & $9(75)$ \\
\hline Read at least part of 2015 ATA guidelines & $4(33)$ & $2(17)$ \\
\hline
\end{tabular}

\section{Barriers to Active Surveillance}

\section{Physician-Related Factors}

Several key barriers to active surveillance emerged at the physician-level, including physician attitudes and knowledge.

\section{Physician Attitudes}

Outcome Expectancy:The most prominent barriers discussed during interviews were related to participants' beliefs about anticipated patient- and disease-related outcomes or consequences of active surveillance. Participants frequently expressed concern about the risk of metastasis if cancer remained in 
place. For example, Surgeon 5 stated, "For my own personal beliefs and biases, I feel that any potential risk for cancer to develop and spread throughout the body is unacceptable." Participants also anticipated experiencing negative emotions, such as guilt, if an adverse outcome occurred during surveillance. Surgeon 1 described, "I would feel uncomfortable...if it grew or spread then I feel like that would be on my shoulders."

In addition, almost half of participants expressed concern that adverse patient outcomes could result from loss of follow-up. They described hesitancy to offer surveillance to unreliable patients because of the potential for cancer progression. For example, Surgeon 6 explained, "it would be safer to [remove] half the thyroid if the patient is never going to come back." Regardless of specialty, participants often viewed surgery as "definitive therapy" (Endocrinologist 10) that provided "certainty" (Endocrinologist 1) and a predictable, expected outcome, whereas surveillance introduced uncertainty.

Some participants also worried that patients would file lawsuits if they experienced an adverse outcome during surveillance. They believed it was important to disclose the potential for poor outcomes prior to initiating surveillance. Surgeon 10 described feeling "legally obligated to say, 'Waiting can allow that cancer a chance to progress."

While many participants described concern about cancer spreading, others acknowledged that metastasis rarely occurs during surveillance. For example, Surgeon 8 stated, "every clinician is afraid they're gonna be surveilling somebody who explodes with lymph node disease...the truth of the matter is that's extremely unlikely to ever happen."

In addition to concern about outcomes, some participants discussed the impact of surveillance on their practice and potential for losing patients and/or referring providers as consequence of not offering thyroidectomy (Figure 2). Even non-surgeons acknowledged patients would seek second opinions and eventually find "a surgeon who would agree to do [surgery]" (Endocrinologist 1).

Agreement:A less prominent barrier to active surveillance was lack of agreement with the guidelines and concern about the concept of watchful waiting. Some participants described being hesitant to recommend surveillance because they are "not treating" (Figure 2) and patients might "feel like we're not caring for them" (Surgeon 6).

Self-Efficacy: Participants also discussed lacking confidence in performing active surveillance. Those who viewed their own lack of experience as a barrier to discussing and offering the approach tended to describe surveillance in vague, as opposed to detailed, terms (Figure 2). Participants also mentioned their inability to convince patients to undergo surveillance because the option is "hard to sell" (Surgeon 1).

Learning Culture \& Motivation: While no participants were unwilling to implement active surveillance in their own practice, many expressed doubts about other physicians' motivation to learn about and incorporate surveillance clinically. Participants believed other physicians are aware of the guidelines, but choose not to follow them or present surveillance as an option (Figure 2). Specifically, some participants 
thought surgeons were not open to surveillance because "they are going to want to cut" (Endocrinologist 6). Meanwhile, surgeons often described thyroidectomy as a "low-risk" option that "provides good results" (Surgeons 7 and 8).

\section{Physician Knowledge}

Awareness \& Familiarity: Few participants expressed concern regarding complete lack of awareness of the guidelines supporting active surveillance. However, many believed lack of familiarity with and/or inappropriate application of the guidelines were barriers. Participants described lag time from guideline release to clinical implementation and expressed that education on how to perform surveillance is lacking (Figure 2).

\section{Guideline-Related Factors}

Barriers to active surveillance that emerged at the guideline-level included lack of evidence, lack of clear intervention goals, and complexity.

Evidence: The most apparent guideline-related barrier was concern about the lack of evidence supporting active surveillance. Many participants believed that data were insufficient and highlighted the lack of prospective trials of patients undergoing surveillance in the US (Figure 3). While some participants discussed trials conducted in Japan as support for active surveillance, several others expressed hesitancy as this data "may not apply to the US population" (Endocrinologist 2).

Intervention Goals: Many participants believed the guidelines lacked clear recommendations and expressed concerns about the goals and duration of active surveillance. Participants described a desire for clear surveillance protocols with more explicit details about patient follow-up, age-specific recommendations, and universal thresholds for nodule progression that prompt further intervention (Figure 3).

Complexity: Participants also discussed that the written guidelines for active surveillance were vague and cited the difficulty and risks of implementing surveillance in their practice without a clear system of patient follow-up in place (Figure 3 ).

\section{External Factors}

Barriers to active surveillance on the external-level focused on social and clinical norms, lack of collaboration, organizational constraints, and lack of resources.

Norms: Barriers related to social and clinical norms were prevalent throughout the interviews, particularly concerns over societal attitudes and beliefs about cancer. All participants discussed the impact of "the $C$ word" on patients' willingness to consider active surveillance (Figure 4). Most described this barrier as resulting from "being in a culture where cancer is bad and needs to get out" (Surgeon 1). 
Participants discussed the influence of clinical norms on patient expectations. Surgeons in particular described how once a patient is referred to a surgeon, it is difficult to get the patient to consider non-surgical management (Figure 4). For example, Surgeon 6 stated, "It'll take a long time for active surveillance to be put into practice from the surgeon's perspective... because [patients] expect surgery when they come to see you."

Collaboration: Participants believed lack of collaboration between specialists poses a barrier to active surveillance. They highlighted the potential for patients to receive conflicting advice about treatment options (Figure 4). Some participants discussed how patient expectations set by referring providers can "create lots of conflict" if their own recommendation does not align with that of the referring provider (Surgeon 3).

Organizational Constraints: Participants also discussed that active surveillance may be feasible on the individual provider-level, but most participants saw their ability to implement surveillance as being limited by lack of organizational infrastructure and their larger practice environment (Figure 4). Organization-level implementation would require "institution-specific protocols for monitoring patients" which can be difficult to put in place (Endocrinologist 5).

Resources: Participants additionally identified numerous resource-related barriers to active surveillance. These barriers included the lack of patient access to ongoing care, lack of skilled community ultrasound technologists, and the increased physician workload that would result from surveilling a large cohort of patients (Figure 4).

\section{Facilitators of Active Surveillance}

While multiple barriers to active surveillance emerged in the interviews, several facilitators were also identified.

\section{Physician-Related Factors}

Physician-related facilitators of active surveillance included positive attitudes about outcome expectancy and self-efficacy as well as knowledge, awareness, and familiarity with the guidelines (Figure 2). Some participants supported the open-ended structure of active surveillance and framed surveillance as a "bridge" to future, more definitive treatment. Most participants identified shared decision-making as another facilitator of surveillance (Figure 2). In terms of physician knowledge, participants described the role of physician education in improving guideline awareness and familiarity and saw continuing medical education as a strategy to increase knowledge about how to perform active surveillance (Figure 2).

\section{Guideline-related factors}

At the guideline-level, clear intervention goals was the only facilitator identified. Nearly all participants described how the goal of the guideline was to avoid surgery and its potential complications, which was a benefit and facilitator of active surveillance (Figure 3). 


\section{External Factors}

Facilitators of active surveillance that emerged at the external-level included social and clinical norms, particularly related to attitudes and beliefs about surgery and the presence of collaboration (Figure 4). Many participants described patients' fear of surgery as a facilitator, citing how surveillance is often the treatment choice for patients who are more afraid of surgery than the possibility of cancer progression. Most participants also expressed that collaboration and a multidisciplinary team approach facilitates guideline implementation (Figure 4).

\section{Discussion}

This qualitative study characterized barriers and facilitators to implementing active surveillance for patients with small, low-risk thyroid cancer in the United States (US) ${ }^{20}$ The most prominent barriers were physician-related. Physicians' beliefs about fear about disease progression and their own ability to offer and perform surveillance contributed to a majority of perceived barriers, while knowledge about guidelines contributed to a lesser extent. Participants also viewed vagueness of surveillance protocols and limited long-term outcome data supporting active surveillance in the US as impeding implementation. Patient expectations and societal beliefs about cancer were also commonly described barriers to implementation. Interventions to address some of these barriers are already underway, including establishing outcomes of active surveillance in US patients $s^{8,9}$, publication of protocols ${ }^{23-27}$, and physician education. Additional interventions should focus on physicians' and patients' beliefs about cancer, facilitating multidisciplinary approaches, and promoting societal-level acceptance of active surveillance.

Physicians' beliefs about the potential negative outcomes of active surveillance was one of the most significant barriers identified. Fear of cancer metastasis and losing patients to follow-up during surveillance appear to hinder physicians from offering surveillance as an option. These fears are reinforced by anecdotal experience and patient expectations about surgery. To effect behavioral change going forward, it will be important to transform physicians' beliefs about the negative consequences of surveillance. Potential interventions include audit-feedback systems, disseminating surveillance protocols, and publishing the data currently being collected on oncologic and patient-reported outcomes. Individualized audit-feedback on patient care decisions has been shown to be effective in other diseases. ${ }^{28,29}$ Multispecialty local- or state-level efforts that incorporate peer coaching may be an alternative strategy to audit-feedback. In other medical settings, coaching has been shown to improve ongoing skills and optimize patient outcomes. ${ }^{30,31}$ These methods could be further supported with educational webinars by national organizations.

Physicians' self-efficacy and beliefs about their ability to offer or perform active surveillance were also prominent barriers. Participants discussed lack of training and proficiency in shared decision-making, limited experience performing surveillance, and being uncomfortable discussing surveillance. Interventions to promote physician confidence include individualized or group training, education, clinical 
decision-making support tools, and reminders that prompt physicians to consider active surveillance. ${ }^{24,32}$ Recent studies in Canada and the US showed that patients would consider less extensive management options if recommended by their physician. ${ }^{16,33-35}$ Therefore, physicians must become comfortable discussing and offering active surveillance as an option. Patient-focused education may also play a role; increasing patients' knowledge and promoting discussion about surveillance could further support acceptance and implementation of this approach. ${ }^{36-38}$

While interventions targeted at physicians and patients are important, overarching societal beliefs must also be addressed. We found that the culturally rooted belief that "you cannot watch cancer" poses a substantial, but not insurmountable, barrier to active surveillance. In the US, active surveillance of low-risk prostate cancer has become an acceptable alternative to active treatment and trials are assessing this approach in women with ductal carcinoma in situ. ${ }^{39}$ Learning from prostate cancer implementation efforts may accelerate acceptance of active surveillance for thyroid cancer. ${ }^{40}$ Strategies to promote societal-level change include national campaigns raising awareness about low-risk thyroid cancer, publishing longitudinal cohort and randomized studies establishing active surveillance safety and outcomes, and developing robust social support networks for patients undergoing surveillance. ${ }^{41}$

Clinical norms related to patient and referring provider expectations of surgical management also pose a barrier to active surveillance. Potentially feasible strategies to reset expectations include local adaptation of guidelines with multidisciplinary collaboration, outreach to community providers, and reframing treatment recommendations. Local adaptation supported by stakeholder groups and task forces enables incorporation of active surveillance into established clinical structures across specialties. ${ }^{42}$ Multidisciplinary collaboration further facilitates acceptance and reduces specialty-specific bias when considering management options. ${ }^{43}$

While this study identified several key barriers and facilitators of active surveillance, it has limitations. First, while we conducted interviews at a national specialty meeting to facilitate physician sample heterogeneity with respect to geography, background, and training, participants' level of knowledge and experience with active surveillance was relatively high compared to most US physicians who treat thyroid cancer. The data were also collected within a year of ATA guideline release. It is possible that some barriers have emerged, changed, or resolved since the data were collected. To address this limitation and ensure the reliability and applicability of our data, we recently performed formal member checking to confirm that the barriers and facilitators described remain relevant and no new barriers have arisen. However, additional barriers may still exist. New data on patient outcomes and protocols for active surveillance have also been published in the interim. ${ }^{4-6,23-26}$ Nonetheless, this study is the first we are aware of to qualitatively highlight the role of physician attitudes and beliefs in adoption of active surveillance in the US.

\section{Conclusion}


This in-depth, qualitative study identified barriers and facilitators of active surveillance of thyroid cancer in the US as perceived by practicing thyroid surgeons and endocrinologists. The most prominent barriers included physician attitudes as well as societal and clinical norms. The findings indicate that adoption of active surveillance can be increased by provider-, patient-, system-, and societal-level interventions. These interventions should address physicians' attitudes and beliefs about the outcomes of surveillance, increase physicians' confidence performing surveillance, and promote societal acceptance of surveillance as a management option

\section{Abbreviations}

ATA, American Thyroid Association; US, United States

\section{Declarations}

Ethics: This study meets the ethics guidelines and adheres to the legal requirements in the United States. The University of Wisconsin Institutional Review Board approved this qualitative study (\#2016-0884).

Consent for publication: Participants provided written informed consent to participate in this study which included the publication of anonymized direct quotations from interviews for publication.

Availability of data and materials: The datasets generated and/or analysed during the current study are not publicly available due to ethical reasons, as they contain information that could compromise the privacy and consent of research participants but are available from the corresponding author (SCP) on reasonable request.

Competing interests: We have no personal conflicts of interest to report and no financial or personal disclosures.

Funding: Support for this research included the American Association of Endocrine Surgeons Paul LoGerfo Research Award and the National Cancer Institute of the National Institutes of Health $(\mathrm{NIH})$ award \#K08CA230204. The content is solely the responsibility of the authors and does not represent the official views of the $\mathrm{NIH}$.

Authors' contributions: Conceived and designed the study: SCP. Collected the data: MCS, SCP. Formally analyzed the data: CBJ, MCS, SCP. Wrote the paper: CBJ, MCS, SCP. Revised and approved the manuscript. CBJ, MCS, SCP. This manuscript has been seen and approved by all authors.

Acknowledgements: We would like to acknowledge Jamia L. Jennings, MS, Huda J. Khokhar, BS, and Esra Alagoz, PhD for their assistance coding and Elizabeth M. Wendt, MD, MPH for assistance interviewing.

\section{References}


1. Haugen BR, Alexander EK, Bible KC, et al. 2015 American Thyroid Association Management Guidelines for Adult Patients with Thyroid Nodules and Differentiated Thyroid Cancer: The American Thyroid Association Guidelines Task Force on Thyroid Nodules and Differentiated Thyroid Cancer.

Thyroid : official journal of the American Thyroid Association. Jan 2016;26(1):1-133. doi:10.1089/thy.2015.0020

2. Ito $Y$, Miyauchi $A$, Inoue $H$, et al. An observational trial for papillary thyroid microcarcinoma in Japanese patients. World J Surg. Jan 2010;34(1):28-35. doi:10.1007/s00268-009-0303-0

3. Sugitani I, Toda K, Yamada K, Yamamoto N, Ikenaga M, Fujimoto Y. Three distinctly different kinds of papillary thyroid microcarcinoma should be recognized: our treatment strategies and outcomes. World J Surg. Jun 2010;34(6):1222-31. doi:10.1007/s00268-009-0359-x

4. Kwon H, Oh HS, Kim M, et al. Active Surveillance for Patients With Papillary Thyroid Microcarcinoma: A Single Center's Experience in Korea. J Clin Endocrinol Metab. 06 2017;102(6):1917-1925. doi:10.1210/jc.2016-4026

5. Molinaro E, Campopiano MC, Pieruzzi L, et al. Active Surveillance in Papillary Thyroid Microcarcinomas is Feasible and Safe: Experience at a Single Italian Center. J Clin Endocrinol Metab. Mar 2020;105(3)doi:10.1210/clinem/dgz113

6. Rosario PW, Mourão GF, Calsolari MR. Active Surveillance in Adults with Low-Risk Papillary Thyroid Microcarcinomas: A Prospective Study. Horm Metab Res. Nov 2019;51(11):703-708. doi:10.1055/a1015-6684

7. Koshkina A, Fazelzad R, Sugitani I, et al. Association of Patient Age With Progression of Low-risk Papillary Thyroid Carcinoma Under Active Surveillance: A Systematic Review and Meta-analysis. JAMA Otolaryngol Head Neck Surg. 06 2020;146(6):552-560. doi:10.1001/jamaoto.2020.0368

8. Banerjee S. Treatment Decision-Making Among Patients Diagnosed With Papillary Microcarcinoma and Their Significant Others. Identifier NCT01392222. https://clinicaltrials.gov/ct2/show/NCT01392222.

9. Ho A. Active Surveillance of Papillary Thyroid Microcarcinoma (PMCAS). Identifier NCT02609685. https://clinicaltrials.gov/ct2/show/NCT02609685.

10. Castro MR, Morris JC, Ryder M, Brito JP, Hay ID. Most patients with a small papillary thyroid carcinoma enjoy an excellent prognosis and may be managed with minimally invasive therapy or active surveillance. Cancer. Sep 2015;121(18):3364-5. doi:10.1002/cncr.29468

11. Tuttle RM, Fagin JA, Minkowitz G, et al. Natural History and Tumor Volume Kinetics of Papillary Thyroid Cancers During Active Surveillance. JAMA Otolaryngol Head Neck Surg. 10 2017;143(10):1015-1020. doi:10.1001/jamaoto.2017.1442

12. Lohia S, Morris LGT, Roman BR. Association Between Implementation of the 2009 American Thyroid Association Guidelines and De-escalation of Treatment for Low-risk Papillary Thyroid Carcinoma. JAMA Otolaryngol Head Neck Surg. Oct 2020;doi:10.1001/jamaoto.2020.3024

13. Roman B, Brito J, Saucke M, et al. National survey of endocrinologists and surgeons regarding active surveillance for low risk papillary thyroid cancer. Endocrine Practice; In press. 
14. Yang N, Saucke M, Marka N, et al. Adoption of Active Surveillance: Analysis of a Large National Cohort of Physicians. Thyroid. 2019;(29(s1):A177-A205)

15. Hughes DT, Reyes-Gastelum D, Ward KC, Hamilton AS, Haymart MR. Barriers to the Use of Active Surveillance for Thyroid Cancer: Results of a Physician Survey. Ann Surg. Oct 2020;doi:10.1097/SLA.0000000000004417

16. Jensen CB, Saucke MC, Francis DO, Voils Cl, Pitt SC. From Overdiagnosis to Overtreatment of LowRisk Thyroid Cancer: A Thematic Analysis of Attitudes and Beliefs of Endocrinologists, Surgeons, and Patients. Thyroid. 05 2020;30(5):696-703. doi:10.1089/thy.2019.0587

17. Esserman LJ, Thompson IM, Reid B, et al. Addressing overdiagnosis and overtreatment in cancer: a prescription for change. Lancet Oncol. May 2014;15(6):e234-42. doi:10.1016/S1470-2045(13)705989

18. Leboulleux S, Tuttle RM, Pacini F, Schlumberger M. Papillary thyroid microcarcinoma: time to shift from surgery to active surveillance? Lancet Diabetes Endocrinol. 11 2016;4(11):933-942. doi:10.1016/S2213-8587(16)30180-2

19. Welch HG, Doherty GM. Saving Thyroids - Overtreatment of Small Papillary Cancers. N Engl J Med. Jul 2018;379(4):310-312. doi:10.1056/NEJMp1804426

20. Fischer F, Lange K, Klose K, Greiner W, Kraemer A. Barriers and Strategies in Guideline Implementation-A Scoping Review. Healthcare (Basel). Jun 2016;4(3)doi:10.3390/healthcare4030036

21. Cabana MD, Rand CS, Powe NR, et al. Why don't physicians follow clinical practice guidelines? A framework for improvement. Jama. Oct 20 1999;282(15):1458-65. doi:10.1001/jama.282.15.1458

22. Birt L, Scott S, Cavers D, Campbell C, Walter F. Member Checking: A Tool to Enhance Trustworthiness or Merely a Nod to Validation? Qual Health Res. Nov 2016;26(13):1802-1811. doi:10.1177/1049732316654870

23. Haser GC, Tuttle RM, Urken ML. Challenges of Active Surveillance Protocols for Low-Risk Papillary Thyroid Microcarcinoma in the United States. Thyroid. 07 2016;26(7):989-90. doi:10.1089/thy.2016.0109

24. Brito JP, Ito Y, Miyauchi A, Tuttle RM. A Clinical Framework to Facilitate Risk Stratification When Considering an Active Surveillance Alternative to Immediate Biopsy and Surgery in Papillary Microcarcinoma. Thyroid. Jan 2016;26(1):144-9. doi:10.1089/thy.2015.0178

25. Sawka AM, Ghai S, Tomlinson G, et al. A protocol for a Canadian prospective observational study of decision-making on active surveillance or surgery for low-risk papillary thyroid cancer. BMJ Open. 04 2018;8(4):e020298. doi:10.1136/bmjopen-2017-020298

26. Patel KN, Yip L, Lubitz CC, et al. The American Association of Endocrine Surgeons Guidelines for the Definitive Surgical Management of Thyroid Disease in Adults. Ann Surg. 03 2020;271(3):e21-e93. doi:10.1097/SLA.0000000000003580

27. Sugitani I, Ito Y, Takeuchi D, et al. Indications and Strategy for Active Surveillance of Adult Low-Risk Papillary Thyroid Microcarcinoma: Consensus Statements from the Japan Association of Endocrine 
Surgery Task Force on Management for Papillary Thyroid Microcarcinoma. Thyroid. Nov 2020;doi:10.1089/thy.2020.0330

28. Ivers N, Jamtvedt G, Flottorp S, et al. Audit and feedback: effects on professional practice and healthcare outcomes. Cochrane Database Syst Rev. Jun 2012;(6):CD000259. doi:10.1002/14651858.CD000259.pub3

29. Joosen MC, van Beurden KM, Terluin B, van Weeghel J, Brouwers EP, van der Klink JJ. Improving occupational physicians' adherence to a practice guideline: feasibility and impact of a tailored implementation strategy. BMC Med Educ. Apr 24 2015;15:82. doi:10.1186/s12909-015-0364-8

30. Greenberg CC, Ghousseini HN, Pavuluri Quamme SR, et al. A Statewide Surgical Coaching Program Provides Opportunity for Continuous Professional Development. Ann Surg. 05 2018;267(5):868-873. doi:10.1097/SLA.0000000000002341

31. Greenberg CC, Ghousseini HN, Pavuluri Quamme SR, Beasley HL, Wiegmann DA. Surgical coaching for individual performance improvement. Ann Surg. Jan 2015;261(1):32-4. doi:10.1097/SLA.0000000000000776

32. Brito JP, Moon JH, Zeuren R, et al. Thyroid Cancer Treatment Choice: A Pilot Study of a Tool to Facilitate Conversations with Patients with Papillary Microcarcinomas Considering Treatment Options. Thyroid. 10 2018;28(10):1325-1331. doi:10.1089/thy.2018.0105

33. Sawka AM, Ghai S, Yoannidis T, et al. A Prospective Mixed-Methods Study of Decision-Making on Surgery or Active Surveillance for Low-Risk Papillary Thyroid Cancer. Thyroid. Jul 2020;30(7):9991007. doi:10.1089/thy.2019.0592

34. Doubleday AR, Saucke MC, Bates MF, Pitt SC. Patient-surgeon decision-making about treatment for very low-risk thyroid cancer. Trends Cancer Res. 2019;14:79-89.

35. Nickel B, Brito JP, Moynihan R, Barratt A, Jordan S, McCaffery K. Patients' experiences of diagnosis and management of papillary thyroid microcarcinoma: a qualitative study. BMC Cancer. 03 2018;18(1):242. doi:10.1186/s12885-018-4152-9

36. Pitt SC, Saucke MC. Novel Decision Support Interventions for Low-risk Thyroid Cancer. JAMA otolaryngology- head \& neck surgery. Sep 24 2020;doi:10.1001/jamaoto.2020.2279

37. Davies $\mathrm{L}$, Chang $\mathrm{CH}$, Sirovich $\mathrm{B}$, et al. Thyroid Cancer Active Surveillance Program Retention and Adherence in Japan. JAMA Otolaryngol Head Neck Surg. Jan 2021;147(1):77-84. doi:10.1001/jamaoto.2020.4200

38. McCaffery KJ, Nickel B. Clinician Be My Guide in Active Surveillance of Papillary Thyroid Microcarcinoma. JAMA Otolaryngol Head Neck Surg. Jan 2021;147(1):7-8. doi:10.1001/jamaoto.2020.4237

39. Cooperberg MR, Carroll PR. Trends in Management for Patients With Localized Prostate Cancer, 1990-2013. Jama. Jul 7 2015;314(1):80-2. doi:10.1001/jama.2015.6036

40. Ho AS, Daskivich TJ, Sacks WL, Zumsteg ZS. Parallels Between Low-Risk Prostate Cancer and Thyroid Cancer: A Review. JAMA Oncol. Apr 1 2019;5(4):556-564. doi:10.1001/jamaoncol.2018.5321 
41. Penson DF. Factors influencing patients' acceptance and adherence to active surveillance. J Natl Cancer Inst Monogr. Dec 2012;2012(45):207-12. doi:10.1093/jncimonographs/lgs024

42. Harrison MB, Légaré F, Graham ID, Fervers B. Adapting clinical practice guidelines to local context and assessing barriers to their use. Cmaj. Feb 9 2010;182(2):E78-84. doi:10.1503/cmaj.081232

43. Aizer AA, Paly JJ, Zietman AL, et al. Multidisciplinary care and pursuit of active surveillance in lowrisk prostate cancer. J Clin Oncol. Sep 1 2012;30(25):3071-6. doi:10.1200/jco.2012.42.8466

\section{Figures}

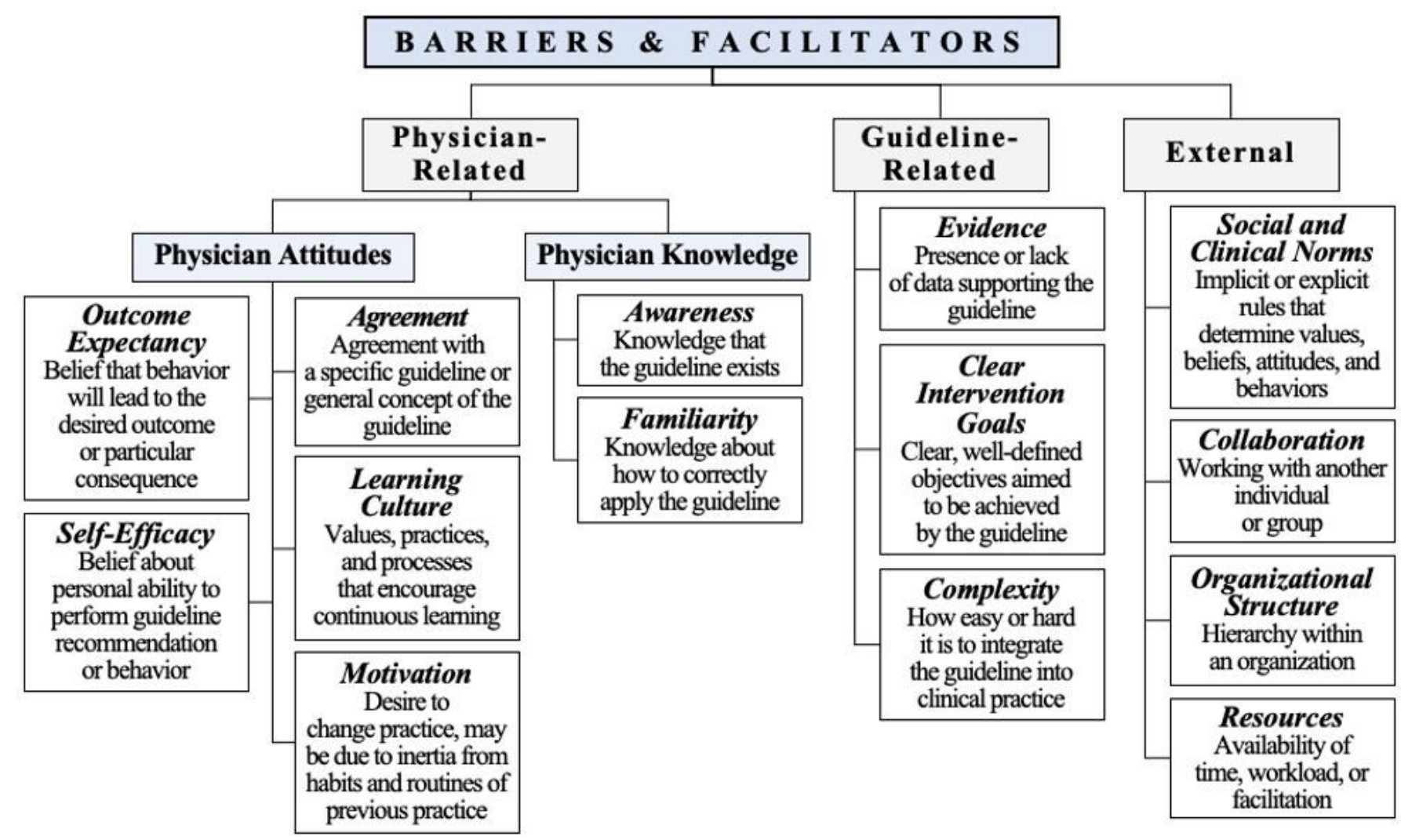

Figure 1

Framework of guideline implementation developed by Fischer et al. categorizes barriers and facilitators to into three domains-physician-related, guideline-related, and external factors. Each barrier/facilitator and its definition are shown here. 
Physician-Related Factors

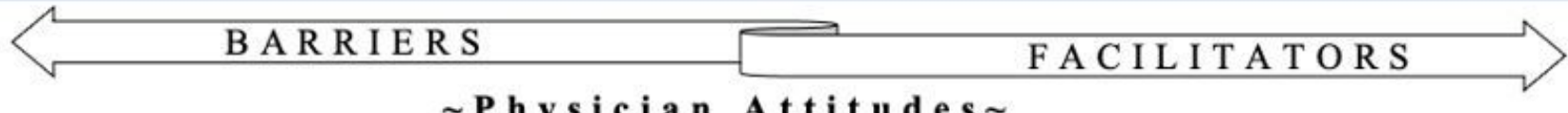

〜Physician Attitudes

"A small percentage of microcarcinomas act aggressively...exceptions to the vast majority. We have to be on guard because our opinions are sometimes colored by these very bad instances." Surgeon 12

"I have lost a couple of referring physicians who basically said, 'I want the whole thing out.' And I say, 'I'm sorry, I can't do it'. " Surgeon 5

"It would be hard for me to not even treat thyroid cancer but treat patients...by watching their cancer. The whole paradigm is very hard for me to buy." Surgeon 1

"Some [clinicians] will not be able to explain [active surveillance]... not just because it's difficult, but because of experience." Endo 2

"I try to impress upon them that as surgeries go, this one is actually not as dangerous as many others...recovery is actually easier." Endo 1
"Some [patients] actually use AS as a bridge...because they just got a new job or their

Outcome daughter's getting married next year." Endo 3

Expectancy

"There's time to make the decision. Go think about this or talk to other people... You can come back to me in six months with a repeat ultrasound. And make the decision again at that point." Surgeon 4

\section{〜Phvsician Knowledge}

"It's also educating physicians in the community...there are tons of general endos that do these and tons of surgeons that do the surgeries." Endo 7
Self-

Efficacy

Learning

Culture \&

Motivation
"The initial conversation is very important ... you set the expectations, give the options, and what will happen if things progress or are stable. As long as [patients] know the process and are fully informed, they are more comfortable...they feel empowered." Endo 12

\section{Figure 2}

Exemplary quotes of physician-related factors as barriers and facilitators to implementing active surveillance (AS) of low-risk thyroid cancer. (Abbreviation: Endo, endocrinologist) 


\section{Guideline-Related Factors}

\section{BARRIERS}

“We're just starting to define the potential for these cancers to grow and spread over time... I don't know if the data are well enough described to make me confident surveilling." Surgeon 5

"If I'm doing active surveillance, I don't have an endpoint...not knowing how long we need to surveil is problematic." Endo 10

"We can lose patients to follow up. We need to be very careful in the US about having a protocol to follow patients." Surgeon 6

\section{F A C I L I T A T O R S}

"There's a well-known series from Japan

Evidence following patients with [thyroid] cancer...a very small percentage progressed or reached an indication for surgery, so that's overall reassuring." Endo 6

Clear Intervention

Goals

Complexity
"The benefit of surveillance is, it's not an operation. [Patients] avoid the relatively low, but real risk of operative complications that could change their quality of life." Surgeon 7

\section{Figure 3}

Exemplary quotes of guideline-related factors as barriers and facilitators to active surveillance of low-risk thyroid cancer. (Abbreviation: Endo, endocrinologist)

\section{External F a ctors}

\section{BARRIERS}

"It's rare that an American patient is happy to live with cancer in their body without treatment. Any chance there's cancer, and the average American will say 'I don't want cancer in my body. '” Endo 9 “...for practice to change, it depends on what doctors say to patients. Patients come to me from other doctors who do not include the fact that they're very good candidates for surveillance." Surgeon 4

"A lot of times endocrinologists who refer cases have already told them you're gonna need to have something done... Those expectations have already been influenced by their referring doc." Surgeon 8

"The average person who lives in the middle of nowhere doesn't have an endocrinologist that can follow them carefully... Logistics... have to be considered." Surgeon 5

"We don't have enough endocrinologists as it is. My clinic is already booked out six months... We're really going to invest human resources even more than financial resources." Endo 10

\begin{tabular}{|c|c|}
\hline & F A C I L I A T O R S \\
\hline & $\begin{array}{l}\text { "...the person that doesn't do surgery is not } \\
\text { the person who is totally comfortable that it's } \\
\text { not gonna grow. It's more of a person who is }\end{array}$ \\
\hline $\begin{array}{r}\text { Social \& } \\
\text { Clinical }\end{array}$ & ...they'd rather watch [the cancer] than go \\
\hline Norms & through surgery." Endo 9 \\
\hline
\end{tabular}

Collaboration "As a group we went through the guidelines, and we outlined what endocrinologists and surgeons were agreeable on pursuing. We tried to be concordant with each other." Endo 7 [on implementing surveillance]

"We have a variety of patients... from a very large metroplex region to rural [state]...We

Organizational Structure

Resources
-1 developed a list of curated imaging centers to follow them on 6-month ultrasound and ... we schedule [follow-up] as per their geographical location." Surgeon 8 
Figure 4

Exemplary quotes of external factors as barriers and facilitators to active surveillance of low-risk thyroid cancer. (Abbreviation: Endo, endocrinologist) 